\title{
TRAUMATIC BONE CYST OF IDIOPATHIC ORIGIN?A REPORT OF TWO CASES
}

\author{
Kumar Satish $^{1}$, S. Padmashree ${ }^{1}$, Jayalekshmy Rema $^{1}$
}

\begin{abstract}
BACKGROUND: Traumatic bone cyst (TBC) is an uncommon non-epithelial lined cavity of the jaws. Traumatic bone cysts have been reported in the literature under a variety of names: solitary bone cyst, haemorrhagic bone cyst, extravasation cyst and simple bone cyst. The multitude of names applied to this lesion implies the lack of understanding of the true aetiology and pathogenesis. However, the term "traumatic bone cyst" is the most widely used name today (1).

CASE DETAILS: A 15 years old male patient presented with a complaint of swelling with mild, intermittent and non-radiating pain in the lower left back teeth region. There was expansion of the buccal cortical plate. Radiograph showed a multilocular lesion straddling between the roots of the teeth. In addition, 21 years old male patient came with a complaint of swelling with mild, continuous pain in the lower left jaw region. OPG showed well defined radiolucency with scalloped borders extending from the periapical region of the $1^{\text {st }}$ premolar until the $2^{\text {nd }}$ molar. The radiolucency was seen extending between the roots of the involved teeth.

CONCLUSION: The majority of TBCs are located in the mandibular body between the canine and the third molar. Clinically, the lesion is asymptomatic in the majority of cases and is often accidentally discovered on routine radiological examination, usually as a unilocular radiolucent area with a "scalloping effect". Since material for histologic examination may be scanty or non-available it is very often difficult for a definite histologic diagnosis to be achieved (1).

KEYWORDS: Traumatic bone cyst, idiopathic origin
\end{abstract}

DOI: http://dx.doi.org/10.4314/ejhs.v24i2.13

\section{INTRODUCTION}

In the classification of the World Health Organization (WHO), TBCs are included in the group of bone-related lesions, together with the aneurysmal bone cyst, ossifying fibroma, fibrous dysplasia, osseus dysplasia, central giant cell granuloma and cherubism. Different causal factors have been proposed: bone tumor degeneration, altered calcium metabolism, low-grade infection, local alterations in bone growth, venous obstruction, increased osteolysis, intramedullary bleeding, local ischemia, or a combination of such factors. It has been suggested that any form of trauma, including tooth extraction could give rise to a cyst of this kind (2).Typically, the lesions are asymptomatic and are accidentally detected by panoramic radiography; they may resolve without treatment (3). The most widely accepted theory suggests bleeding within the bone is caused by trauma. Instead of the organization of a blood clot and healing, the clot liquefactively necrotises or is resorbed in another way. The surrounding bone is destroyed by enzymatic activity. In this way the bone cavity enlarges, stimulated by the increased pressure of its content, which, at least partly, is caused by poor venous drainage (1). An investigation of 255 cases during the period 1955-

\footnotetext{
${ }^{1}$ Oral Medicine and Radiology, Vydehi Institute of Dental Science and Research Center, Bangalore, Karnakata, India
} 
1979 showed that TB occurred in the mandible in $89 \%$, while it occurred in the maxilla in only $11 \%$. In the mandible, only $25 \%$ of TBC occurred in the frontal segment, while as many as $67 \%$ occurred in the frontal part of the maxilla. Thus, TBCs occurs frequently in the mandible, mainly in the posterior part, while in the maxilla, they are rare and mainly located in the frontal part (3). The present study describes the clinical and radiological characteristics, and the surgical findings of traumatic bone cysts in two patients.

\section{CASE REPORT 1}

A 15 years old male patient presented with a complaint of mild, intermittent and non-radiating pain in the lower left back teeth region since one year. Pain occurs on chewing hard food.

On extra oral examination, a diffuse swelling roughly oval in shape measuring around $2 \mathrm{~cm}$ in diameter was seen on the lower left jaw region (Fig1). Intraorally, there was obliteration of the buccal vestibule i.r.t. $35,36,37$. There was expansion of the buccal cortical plate. Considering the clinical findings, provisional diagnosis of ameloblastoma and differential diagnosis of odontogenic keratocyst (OKC) and central giant cell granuloma (CGCG) were considered.

Intraoral periapical radiograph i.r.t. 36, 37 showed was multilocular radiolucency with thin septa extending from the middle half of the root to

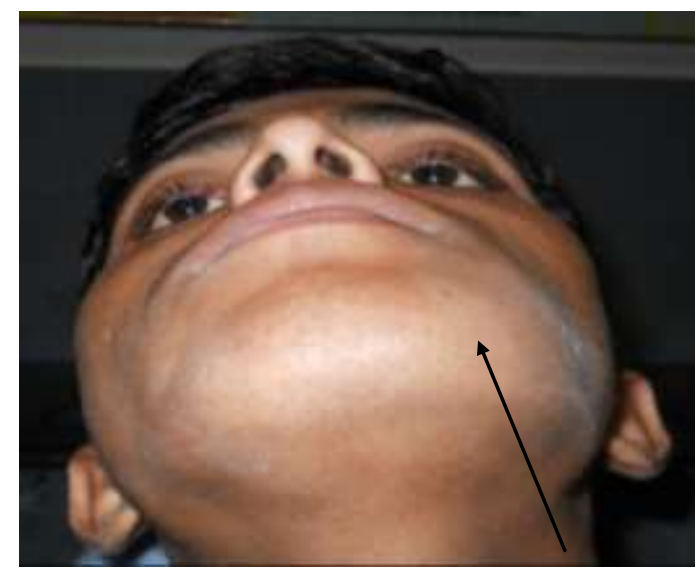

Fig 1: Extra oral view showing swelling in relation to the lower left mandibular region. the periapices of the teeth (Fig 2). Mandibular occlusal radiograph showed expansion of the buccal and the lingual cortical plate. OPG showed a multilocular lesion straddling between the roots of the teeth involving the body, angle and ramus of the mandible on the left side. Wispy septa could also be seen. No root resorption or displacement of the teeth was seen. There was thinning of the inferior border of the mandible (Fig 3). The radiological findings suggested a differential diagnosis of traumatic bone cyst and CGCG.

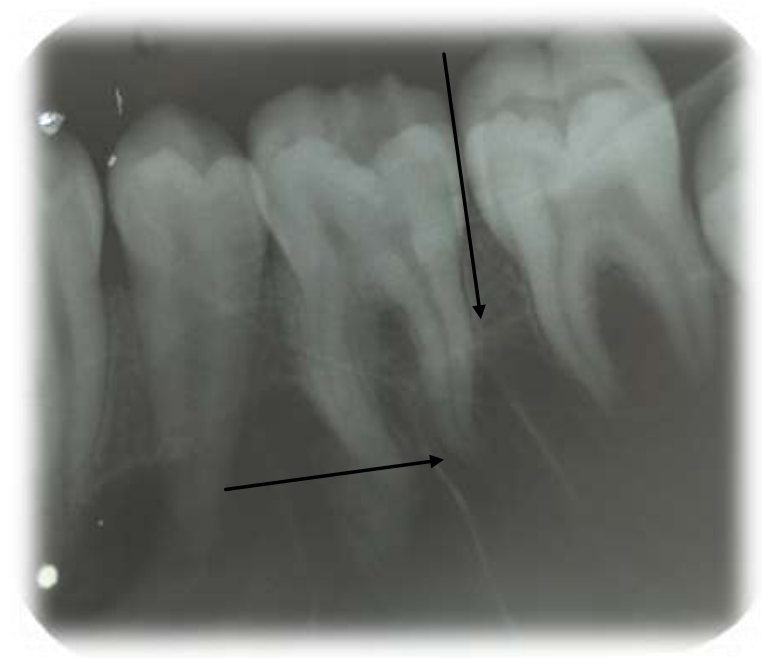

Fig 2: Intraoral radiograph i.r.t. 36 and 37 showing scalloped, multilocular radiolucency.

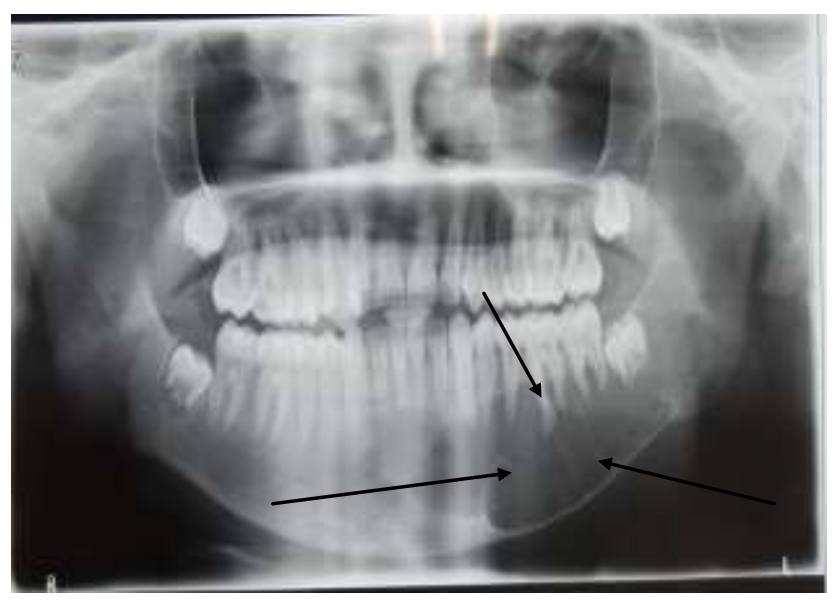

Fig 3: OPG showing multilocular radiolucency in the left mandibular region extending mesially from the midline till distal of 38 . 
Haematological investigations revealed elevated serum Alkaline phosphatase-221 IU/L. Serum calcium-9.7 mg/dl and Serum phosphorus$4.2 \mathrm{mg} / \mathrm{dl}$ were within normal limits. Serum potassium and chloride levels were high with values of $6.3 \mathrm{mEq} / \mathrm{L}$ and $110.7 \mathrm{mEq} / \mathrm{L}$ respectively. On surgical exploration, a hollow bone cavity filled with blood was seen confirming the lesion to be as traumatic bone cyst (Fig 4). H \& E stained decalcified section shows delicate fibrous connective tissue lining with capillaries and areas of hemorrhage. Few trabeculae can be seen at the periphery. These features are suggestive of traumatic bone cyst.

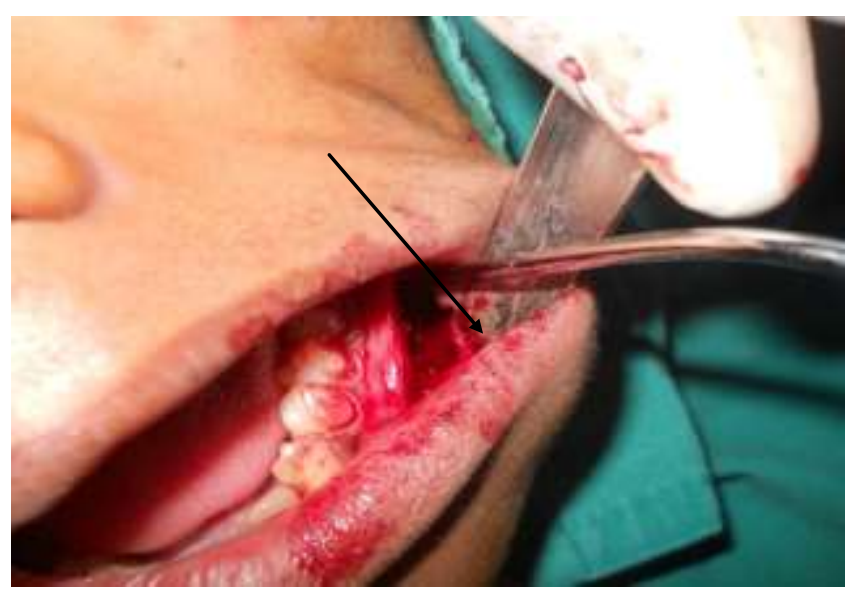

Fig 4: Biopsy site revealing blood filled cavity.

Considering the clinical, radiological and histopathological findings and the extent of the lesion with thinning of inferior cortical border of the mandible, segmental resection of the mandible with free vascularised fibular reconstruction was done.

\section{CASE REPORT 2}

A 21 year old male patient came with a complaint of mild, continuous pain in the lower left jaw region that lasted for one week. On intraoral examination, a swelling measuring around $2 \mathrm{~cm}$ was seen in the gingival area extending from the $1^{\text {st }}$ premolar till the distal of $2^{\text {nd }}$ molar (Fig 5). The swelling was hard in consistency and non-tender on palpation. The associated teeth were not decayed and non-tender on percussion. Vitality tests showed that the associated teeth were vital. OPG showed well defined radiolucency with scalloped borders extending from the periapical region of $1^{\text {st }}$ premolar till $2^{\text {nd }}$ molar. The radiolucency was seen extending between the roots of the involved teeth. No root resorption or displacement of teeth was seen (Fig 6). Considering the clinical and radiological findings, a provisional diagnosis of traumatic bone cyst was considered. Surgical exploration of the involved area revealed an empty cavity filled with blood. The body cavity has been sutured back after surgical exploration.

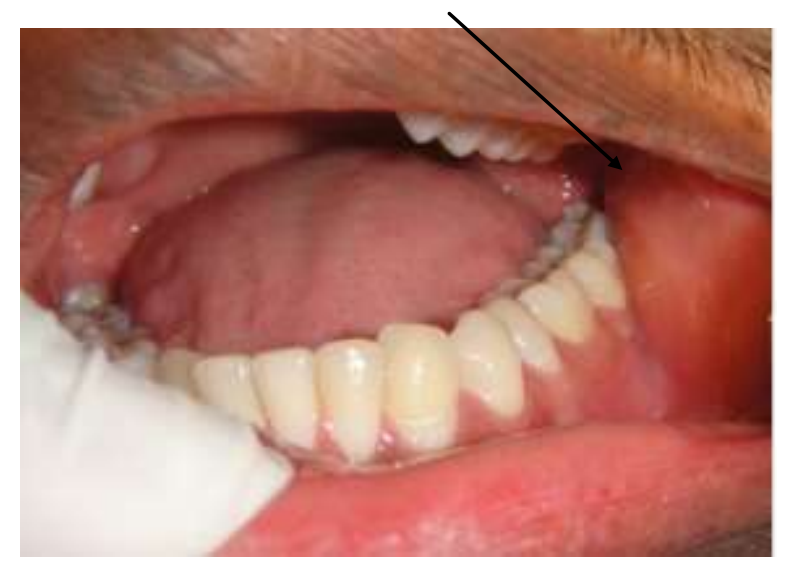

Fig 5: Intraoral view of the lower left buccal vestibule.

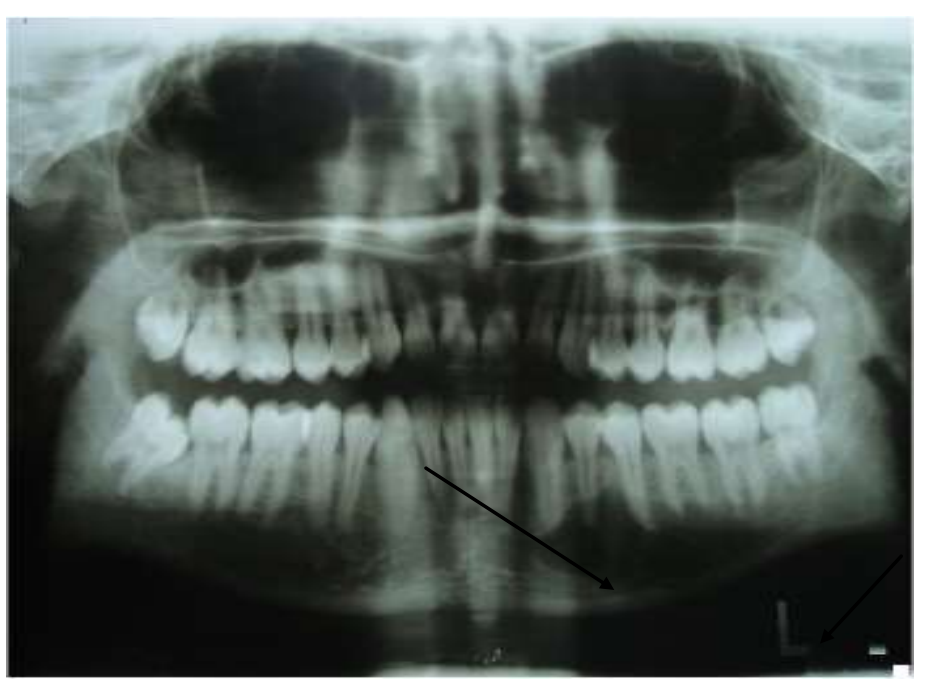

Fig 6: OPG showing well defined radiolucency extending from mesial of 33 till distal of 37 extending between the roots of the teeth.

\section{DISCUSSION}

The pathogenesis of the TBC still remains a matter of conjecture; several theories have been 
suggested. Trauma is the most frequently discussed etiologic factor in the formation of a TBC. The presence of a history of trauma is extremely variable in the reported series of cases from $17 \%$ to $70 \%$ (1). Trauma leads to intraosseous hematoma formation. After trauma, the blood clot liquefies, and adjacent bone is destroyed by enzymatic activity. Blum and Thoma believed that a previous traumatic episode to the jaws contributes to the development of most of TBCs $(4,5)$. Thoma suggested that trauma initiates a subperiosteal hematoma that causes a compromised blood supply to the area, leading to osteoclastic bone resorption (5). Traumatic bone cysts are generally detected in patients in the second and third decades of life, though they can also be found in older age groups (6). The mandibular body is the frequent site of TBC; however, cases involving the mandibular symphysis, ramus, condyle and even anterior maxilla have been reported $(7,8,9,10)$.

Clinically, the soft tissues are without any change. There is no increased mobility of the teeth or changes in their color. The teeth are rarely sensitive to percusion. There is rarely a sensation of pain or even paresthesia. The blood count is unchanged. There is usually no bone expansion, particularly in the early phases, while in the later phase; it is possible to perceive bone expansion in $18-50 \%$ of patients. It can be said that the asymptomatic nature of the disease is the reason why TBC is occasionally detected accidentally on a radiograph, performed for completely different reasons and because of other diseases (2).

On imaging, the TBC appears as a radiolucent area with scalloped borders and sometimes with slight cortication. Scalloping of the border was prominent in the present cases; this feature is suggestive, but not diagnostic, of TBC, because several other odontogenic lesions have scalloped borders. Compete et al. reported scalloping pattern in $68 \%$ of TBCs occurring between and away from teeth(11).

Most of the cases are asymptomatic and are diagnosed in the routine radiological investigations. The lamina dura may or may not be lost, and occasionally root resorption is seen. Treatment of solitary bone cysts is surgical exploration and curettage of the bone wall. However, the recurrence rate was found to be greater than $20 \%$. In most cases, healing or recurrence will be confirmed within 3 years after surgery (2).

Regarding the first case, segmental resection with free vascularised fibular reconstruction was done as there was extensive loss of bone with only the inferior border of the mandible intact which could lead to pathological fracture. The second case had a less extensive lesion. The associated teeth were vital and non-tender on percussion. No associated mobility of the teeth was noted. Radiograph showed a multilocular lesion straddling between the roots of the teeth. This is suggestive of a traumatic bone cyst. Surgical exploration of the cavity along with inducing of bleeding points was done.

In conclusion, although it is called traumatic bone cyst, a clear history of trauma is highly variable (11). The importance of trauma in the development of TBCs may thus appear questionable as seen in the two cases discussed above. However, of the pathogenic hypothesis of TBCs, the most widely accepted mechanism involves microtrauma and subsequent intramedullary bleeding, with osteoclastic activity. This leads to elimination of trabeculae within the cancellous bone compartment, thus giving rise to a cystic cavity (4). Asymptomatic bony swelling of the mandible in a young patient with or without history of trauma should raise the suspicion of a traumatic bone cyst so that prompt intervention can be made.

\section{REFERENCES}

1. Arsinoi A Xanthinaki, Konstantinos I Choupis et al. Traumatic bone cyst of the mandible of possible iatrogenic origin: a case report and brief review of the literature. Head \& Face Medicine. 2006; 2:40.

2. Sebastijan Sandev, Klara Sokler, Jonko Grgurevie. Traumatic bone cysts. Acta Stomat Croat, 2001; 35: 417-420.

3. P. F. Perdigão, E. C. Silva, E. Sakurai, N. Soares de Araújo, R. S. Gomez. Idiopathic bone cavity: a clinical, radiographic, and histological study. British Journal of Oral and Maxillofacial Surgery, 2003; 41: 407409.

4. Blum T: Additional report on traumatic bone cysts. Oral Surg Oral Med Oral Pathol, 1955; 8(9):917-39. 
5. Thoma KH: A symposium on bone cysts (editorial). Oral Surg, 1955; 8:899-901.

6. Isidoro Cortell-Ballester, Rui Figueiredo, Traumatic bone cyst: A retrospective study of 21 cases Med Oral Patol Oral Cir Bucal. 2009 May 1;14 (5):E239-43

7. Howe GL: "Haemorrhagic cysts" of the mandible. Br J Oral Surg, 1965; 3:55-91.

8. Hansen L, Sapone J, Sproat R: Traumatic bone cysts of jaws. Report of sixty-six cases. Oral Surg; 1974; 37:899-910.
9. Huebner G, Turlington E: So-called traumatic (hemorrhagic) bone cysts of the jaws. Oral Surg, 1971; 31:354-365.

10. Beasley JD: Traumatic cyst of the jaws: report of 30 cases. J Am Dent Assoc, 1955; 92:145-152.

11. Kuttenberger J, Farmand M, Stoss $\mathrm{H}$ : Recurrence of a solitary bone cyst of the mandibular condyle in a bone graft. Oral Surg Oral Med Oral Pathol, 1992;74:550556. 